\title{
Determination of Bioequivalence for Drugs with Narrow Therapeutic Index: Reduction of the Regulatory Burden
}

\author{
Laszlo Endrenyi ${ }^{1}$ and Laszlo Tothfalusi ${ }^{2}$ \\ ${ }^{1}$ University of Toronto, Department of Pharmacology and Toxicology, Toronto, ON, Canada; ${ }^{2}$ Semmelweis University, \\ Department of Pharmacodynamics, Budapest, Hungary
}

Received, October 21, 2013; Revised, November 16, 2013; Accepted, November 19, 2013; Published, November 22, 2013

\begin{abstract}
The US Food and Drug Administration (FDA) has recently suggested that the bioequivalence (BE) for products of drugs with narrow therapeutic indices (NTI) be assessed by the approach of referencescaled average BE (SABE). Subsequently, in December, 2012, the FDA issued draft guidances for the comparison of products of warfarin sodium and of tacrolimus. The guidances expect that 4-period studies be performed, that the results be evaluated by SABE, and that the analysis include also unscaled average BE as well as the comparison of the estimated within-subject variations $\left(\mathrm{s}_{\mathrm{W}}\right)$ of the test and reference drug products. This communication discusses the new guidances and suggests considerations to reduce the regulatory burden. It is demonstrated that $\mathrm{SABE}$ could be applied when the within-subject variation of the reference product is not higher than $21.42 \%$. Beyond this variation, the BE limits would remain $80 \%$ to $125 \%$, as usual. No further testing by unscaled average $\mathrm{BE}$ is needed. It is also suggested that a comparison of the within-subject variations of the two drug products although interesting for both NTI and other drugs, is not essential for the determination of BE. In addition, when the within-subject variabilities are low then their ratio depends mainly on the nonproduct dependent factors. Moreover, introduction of an additional test would affect the probabilities involved in the primary comparison of the two means. Therefore, the test of comparing variances is not needed and replicate measurements of the test formulation need not be performed. Alternative considerations and approaches, including the use of partial AUC's, are suggested for the determination of BE for NTI drugs.
\end{abstract}

This article is open to POST-PUBLICATION REVIEW. Registered readers (see "For Readers") may comment by clicking on ABSTRACT on the issue's contents page.

\section{INTRODUCTION}

The Food and Drug Administration (FDA) published recently a new draft guidance on the determination of bioequivalence of warfarin sodium formulations (1). The new guidance has a number of new and even novel features. This is interesting to potential sponsors of bioequivalence (BE) studies and to those interested in regulatory sciences. Subsequently, another draft guidance was issued on the bioequivalence of tacrolimus formulations (2). It referred for the methodology to be applied to the draft guidance on warfarin (1).

The new features include the application of the approach of scaled average bioequivalence (SABE) to the bioequivalence of warfarin or tacrolimus products and, by implication, to preparations of drugs with narrow therapeutic indices (NTI) (3). The new warfarin draft guidance presents, for the first time, also a (disaggregated) criterion for the comparison of within-subject variations. These features will be described later in greater detail.

There is no strict definition of what is an NTI (or critical-dose) drug. A general definition is, as $\mathrm{Yu}$ noted, that with NTI drugs "small differences in dose or blood concentration may lead to serious therapeutic failures and/or adverse drug reactions" (4). Warfarin is an NTI drug for which several investigations compared the effects of different products. Reviews of studies noted that brand-name and most generic products had closely similar bioavailabilities and clinical outcomes (5-7). Nevertheless, caution was often offered in studies

Corresponding Author: Laszlo Endrenyi, University of Toronto, Department of Pharmacology and Toxicology, 1 King's College Circle, Toronto, ON, M5S 1A8, Canada. E-mail: 1.endrenyi@utoronto.ca 
and editorials against switching among warfarin products.

Tacrolimus is also an NTI drug $(2,8,9)$. Its various formulations were found to be bioequivalent in healthy subjects (e.g., 10-13). However, concern was expressed about the scarcity of BE data in transplant patients (14).

The question arises if, with the new features of the warfarin draft guidance, the determination of BE for NTI drug products is implemented most advantageously. The purpose of this communication is to explore some answers to this question and to suggest alternative possibilities.

First, relevant aspects of the new warfarin draft guidance will be summarized. This will be followed by an exploration of some of its quantitative features. Comments and recommendations will be presented of alternatives which may have benefits of reduced regulatory burden and enhanced simplicity.

\section{BACKGROUND}

\section{Draft Guidance on the Bioequivalence of Warfarin Sodium Products}

The procedures proposed in the recent warfarin draft guidance (1) will be briefly described together with relevant background information when necessary.

The draft guidance suggests that fully replicated 4-way crossover in vivo studies be performed on healthy males and nonpregnant females chosen from the general population. The studies are to be undertaken under both fasting and fed conditions. Preparations of $10 \mathrm{mg}$ tablets of warfarin sodium are to be administered orally.

The method of reference-scaled average bioequivalence (SABE) should be applied for the statistical comparison of the relevant pharmacokinetic parameters. The approach has been proposed to evaluate the BE of highly-variable drugs and drug products (15). FDA has adopted and implemented it $(16,17)$. The procedure modifies the usual requirement for the determination of BE. Typically, the means $(\mu)$ of a test $(T)$ and a reference $(\mathrm{R}]$ formulation are compared following the logarithmic transformation of the data. It is expected for (unscaled) average $B E$ that the two $90 \%$ confidence interval around the difference between the two logarithmic means be within the preset bioequivalence limits (BEL):

$$
-\mathrm{BEL} \leq \mu_{\mathrm{T}}-\mu_{\mathrm{R}} \leq \mathrm{BEL}
$$

BEL is usually set at the value of $\ln (1.25)$. Consequently, the usual regulatory requirement is, by applying the approach of (unscaled) average BE, that the $90 \%$ confidence interval around the ratio of the geometric means between the two drug products be within the range of 0.80 to 1.25 .

In the approach of scaled average $B E$, the difference between the logarithmic means is standardized by the within-subject standard deviation of the reference formulation $\left(\sigma_{\mathrm{WR}}\right)$ :

$$
-\theta_{\mathrm{A}} \leq\left(\mu_{\mathrm{T}}-\mu_{\mathrm{R}}\right) / \sigma_{\mathrm{WR}} \leq \theta_{\mathrm{A}}
$$

The bioequivalence limits for the scaled, SABE procedure $\left(\theta_{\mathrm{A}}\right)$ and the unscaled average $\mathrm{BE}(\mathrm{BEL})$ are related by:

$$
\theta_{\mathrm{A}}=\ln (\mathrm{BEL}) / \sigma_{\mathrm{W} 0}
$$

Here $\sigma_{w_{0}}$ is a constant the value of which is set by the regulatory authorities.

The draft guidance on the $\mathrm{BE}$ of warfarin products recommends $\mathrm{BEL}=1 / 0.9=1.11111$ and $\sigma_{\mathrm{W} 0}=0.10$ (1). Consequently, the scaled average BE limit is $\theta_{\mathrm{A}}=1.054$.

\section{Steps of Statistical Analysis Recommended by the Draft Guidance}

The steps presented by the draft guidance will be briefly summarized.

Step 1. Calculate the estimated withinsubject standard deviation of the reference product $\left(s_{W R}\right)$, from the results of the completed study, by the usual procedure applied to a (balanced) replicate design.

Step 2. Apply the procedure of referencescaled average BE (SABE) to each pharmacokinetic parameter. Evaluate the upper 95\% confidence bound to the linearized, squared and rearranged form of SABE:

$$
\left(\mu_{\mathrm{T}}-\mu_{\mathrm{R}}\right)^{2}-\theta_{\mathrm{A}}^{2} * \sigma_{\mathrm{WR}}^{2} \leq 0
$$

By inserting estimated values of the means and the variance, and their distributions, the approximate confidence bound can be calculated $(18,19)$ following the approach of Howe (20).

Step 3. By using unscaled average BE, evaluate, for each pharmacokinetic parameter, the $90 \%$ confidence interval around the ratio of geometric means of the two drug products. The $90 \%$ confidence limits should be, as usual, between 
0.8000 and 1.2500 .

Step 4. Evaluate the upper limit of the $90 \%$ confidence interval for the ratio of within-subject standard deviations of the two drug products. The ratio of the variances is characterized by an Fdistribution. The calculated $90 \%$ upper limit should not be higher than 6.25 , i.e., the estimated upper limit for the ratio of the standard deviations should not exceed 2.5.

\section{POSSIBLE REDUCTIONS OF THE REGULATORY BURDEN}

\section{Confidence Limits for Unscaled Average BE}

It will be useful to consider scaled average BE in a corresponding but different form. Multiplying the defining equation [2] by $\sigma_{\mathrm{WR}}$ yields:

$-\theta_{\mathrm{A}} * \sigma_{\mathrm{WR}} \leq \mu_{\mathrm{T}}-\mu_{\mathrm{R}} \leq \theta_{\mathrm{A}} * \sigma_{\mathrm{WR}}$

The expression characterizes unscaled average bioequivalence, as in Eq. 1, but with expanding limits (ABEL) $(19,21)$. The limits are proportional to the within-subject standard deviation $\left(\sigma_{\mathrm{WR}}\right)$.

The approach of ABEL has the advantage over the procedure of SABE that it can be easily assessed by Schuirmann's two one-sided tests method (22). The expanding limits can be calculated by using the values for BEL $=1.11111$ and $\sigma_{\mathrm{W} 0}=0.10$ suggested by the draft guidance for warfarin. The limits are illustrated against increasing within-subject variation in Figure 1. (The diagram displays the variation in terms of the intrasubject coefficient of variation.) Similar figures were shown at a meeting of the FDA Advisory Committee but without the interpretation given here (23-26).

As expected, the implied BE limits are seen to expand with increasing variation. However, it is not desirable that the BE limits be wider than the customary range of 0.80 to 1.25 . This is the intent of Step 3 of the draft guidance. Thus, above a certain variation, the widening of the BE limits should stop. This is also illustrated in Figure 1. The figure demonstrates that the widening stops at a coefficient of variation of $21.42 \%$ beyond which the BE limits remain constant.

It can thus be concluded that the approaches of SABE and ABEL may be applied if, and only if, the within-subject variation of the reference product does not exceed $21.42 \%$. Therefore it is sufficient to estimate the value of $\sigma_{\mathrm{WR}}$ and see if it is below or above 0.2118 (corresponding to $\mathrm{CV}=21.42 \%$ ). No

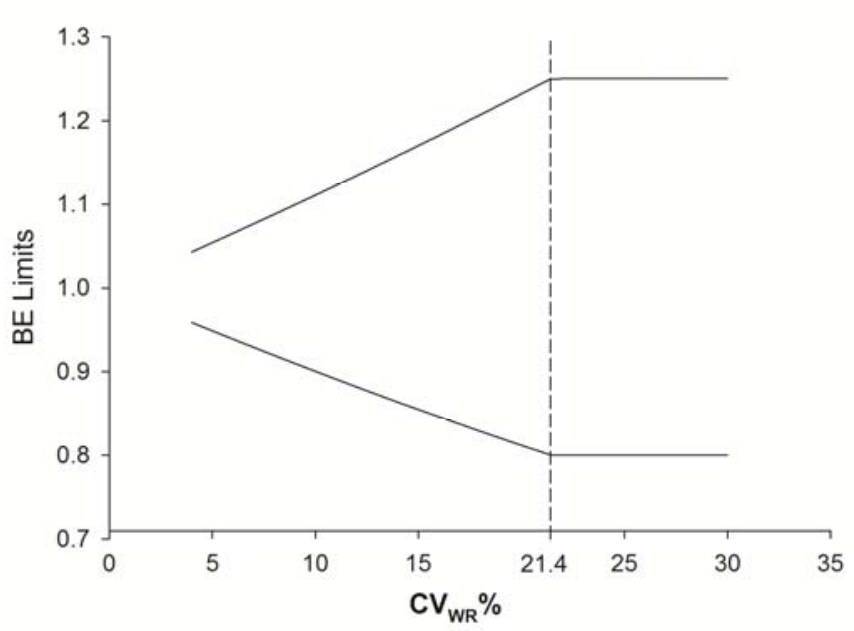

Figure 1. Implied bioequivalence limits for the comparison of NTI drug products. With increasing within-subject variation of the reference product, the BE limits widen until $\mathrm{CV}=21.42 \%$. The limits remain constant at 0.80 and 1.25 at higher variations.

additional test is needed to demonstrate the separation of regions where either scaled or unscaled average BE should be evaluated. Consequently, Step 3 of suggested testing procedure is superfluous and unnecessary.

\section{Determination of the Within-Subject Variation of Both Drug Products}

The warfarin draft guidance suggests that the within-subject variations of not only the reference formulation but also of the test product be estimated $\left(\sigma_{\mathrm{WR}}\right.$ and $\sigma_{\mathrm{WT}}$, respectively). This can be accomplished, as the draft guidance recommends (1), if both formulations are measured twice in each subject.

As noted earlier, the draft guidance expects that the two variations be compared, in Step 4, by an F-test. This is a new requirement in the area of evaluating BE studies. It is not clear that it is needed any more for NTI drugs than for drugs not in this category.

The within-subject variations of drugs and drug products are interesting. These data are always "nice to know". However, it not clear that they are essential in determinations of BE for either NTI or other drugs. Similarly, placing a cap on the deviation between the variations observed for the two formulations is potentially attractive. However, it is not more important for NTI drugs, notably for warfarin and tacrolimus, than for other drugs. 
Therefore it is suggested that it is not necessary to replicate measurements of the test product within individuals. Also, Step 4 suggested in the warfarin draft guidance (1) is not needed.

Two additional comments are offered in regard to the comparison of variances in BE studies. First, the proposal was offered and discussed extensively earlier in connection with the evaluation of individual and population bioequivalence (27, 28 ). At that time, aggregated regulatory criteria were considered. The warfarin draft guidance presents a disaggregated regulatory expectation.

Secondly, introduction of a second regulatory criterion, that for the comparison of variances, would affect and alter the features for the errors of the principal criterion, that for the comparison of the means. This aspect is of concern and should be further investigated.

\section{DISCUSSION}

\section{Are Separate Estimates of Within-Subject Variations Needed for NTI Drug Products?}

It is questionable that the comparison of $\sigma_{\mathrm{WR}}$ and $\sigma_{\mathrm{WT}}$ is meaningful if they are low which is typical for NTI drugs. The within-subject variation is a net effect of errors from many different sources. When $\mathrm{CV}_{\mathrm{WR}}$ is low (say, less than 20\%) then error sources include, first, the bioanalytical error. The bioanalytical guidelines $(29,30)$ require only that the precision determined at each concentration level should not exceed $15 \%$ except at the lower limit of quantitation (LLOQ) at which it should not be higher than $20 \%$. Thus, the bioanalytical error component can be quite substantial, particularly for $\mathrm{C}_{\max }$. Other potential and often neglected error sources are the differences between the actual and nominal contents of active ingredients and the within-batch variations.

Furthermore, warfarin is a BCS Class 1 drug (31). Tablets of warfarin have uncomplicated technology and its bioavailability is nearly complete. If warfarin were not an NTI drug then its bioavailability could be decided based on in-vitro tests. Therefore, it is questionable that screening for product-related specific differences between $\sigma_{\mathrm{WR}}$ and $\sigma_{\mathrm{WT}}$ is a reasonable approach for warfarin. Thus, separate estimates of $\sigma_{\mathrm{WR}}$ and $\sigma_{\mathrm{WT}}$ may not be needed for low-variability BCS Class 1 drugs.

\section{Consistency between Regulatory Requirements}

There are many regulatory requirements which should be met by generic products to get approval.
For instance, the United States Pharmacopeia (USP) specifies for content variability that 10 tablets from any batch must contain $85 \%$ to $115 \%$ of the labelled strength, with a standard deviation of less than $6 \%$. This requirement is more liberal than the proposed bioequivalence criterion. Thus, a situation could arise that allowed the tablet-to-tablet difference of the reference product to be potentially larger than the allowed difference between the test and reference formulations. This is counterintuitive and forces to rethink the consequences of changing only one of the regulatory rules. Stability requirements could be another, similar issue. Thus, bioequivalence expectations should be set in harmony with other quality requirements in order to avoid inconsistencies in the regulatory framework. For warfarin generics, producers often apply stricter content variability standards than the originator (32). It is not clear that such stricter criteria are general and applicable in other cases.

\section{Alternative Approaches}

It is still debatable whether a specific regulation is needed for the bioequivalence of NTI drugs. There are only sporadic clinical reports about negative consequences of switching. Hard evidence in the form of randomized clinical trials is not available and not even expected to be available in the near future. But using data of a small bioequivalence trial with carbamazepine (CBZ), which is an NTI drug, it was demonstrated that the concern is not imaginary. Two have been offered which could support the cautionary approach to generic switching with this drug $(33,34)$. First, it has been reported that the relative risk of neurological adverse effects increases by as much as $50 \%$ due to switching between two CBZ formulations which were otherwise bioequivalent. In that case, $\mathrm{C}_{\max }$ was not a sensitive metric which would be able to capture clinically important differences. As a remedy, the use of partial AUC was suggested for assessing the bioequivalence of CBZ tablets (33).

A second explanation is based on the observation that the assumption that bioequivalence results obtained in a healthy population in singledose studies can be extrapolated to the target patient population at the steady state, is not always true (34). CBZ is a strong enzyme inducer (35) and the $\mathrm{C}_{\max }$ and $\mathrm{C}_{\min }$ concentrations depend on the clearance. A small difference between $\mathrm{C}_{\max }$ values in a healthy population can be significantly higher in the target group. Two formulations which are bioequivalent in a single-dose study with healthy 
volunteers may not be bioequivalent at steady state in patients.

Neither of these problems, the inadequate metric and/or inadequate study population, is addressed directly by the proposed new regulation. Instead, it focuses on the presumed problem of variation difference between the test and reference formulations. It remains to be demonstrated that this is a real clinical problem or just an interesting statistical concept.

\section{CONCLUDING REMARKS}

The procedure in the new draft guidance of FDA on the $\mathrm{BE}$ of warfarin sodium products is remarkably innovative (1). Most importantly, it introduces the application of the scaled average BE procedure to the $\mathrm{BE}$ of formulations of a drug having a narrow therapeutic index. Another example is the new draft guidance on the comparison of tacrolimus formulations (2).

The present communication recognizes the main feature of the new draft guidances, the introduction of the procedure of scaled average $\mathrm{BE}$ for certain NTI drugs. At the same time, suggestions are offered in order to reduce the regulatory burden.

The first recommendation is simple and straightforward. It is not necessary to calculate the $90 \%$ confidence limits with unscaled average BE (Step 3 in the draft guidance) in order to ascertain if the limits obtained with SABE would or would not exceed the 0.80 to 1.25 range. If the estimated within-subject variation of the reference product does not exceed $21.42 \%$ then, automatically, the unscaled average $\mathrm{BE}$ limits are not penetrated.

The second suggestion observes that FDA introduces a new, additional regulatory criterion comparing, by an F-test, the within-subject variances of the two formulations and placing a cap on their ratio. This information is interesting but not more important and essential for comparing formulations of NTI drugs than of other drugs. It is further noted that the additional regulatory criterion modifies the statistical features of the primary criterion that of comparing the means. Therefore it is suggested that Step 4 described in the warfarin draft guidance (1) is not needed. Also, it is not necessary to obtain replicate measurements of the test formulation within individuals.

The suggestions and remarks in the present communication arise from and immediately apply to the recently issued FDA draft guidances on the
BE of warfarin sodium and tacrolimus products (1, 2). However, the agency indicated that it intends to apply the approach of scaled average BE, more generally, to products of drugs having narrow therapeutic indices $(3,25,36)$. The Advisory Committee on Pharmaceutical Science and Clinical Pharmacology supported this intention (37). Thus, our suggestions and remarks may have wider relevance.

It is often assumed that NTI drugs have small within-subject variations $(3,38)$ and that the assessment of $\mathrm{BE}$ for their products should involve narrower than the usual BE limits. The assumption of small intrasubject variation is not always valid. For example, for dalbigatran, in a replicate-design study, the geometric CVs of the two formulations for AUC were $62.2 \%$ and $44.3 \%$, and for $\mathrm{C}_{\max }$ $64.7 \%$ and $45.5 \%$ (39).

As a consequence of this assumption, regulatory guidances call for narrower bioequivalence limits by, for instance, EMA, Health Canada and, now, FDA $(2,3,8,40)$. However, questions can be raised about this regulatory expectation. For instance, alternative metrics such as partial AUC, could provide a more effective approach for dealing with the issue of BE for NTI products (33).

\section{ACKNOWLEDGMENT}

We appreciate the perceptive comments of Dr. Yu Chung Tsang of Apotex on an earlier version of this manuscript.

\section{REFERENCES}

1. US Food and Drug Administration. Center for Drug Evaluation and Research (CDER). Draft guidance on warfarin sodium. December, 2012. http://www.fda.gov/downloads/Drugs/GuidanceCompli anceRegulatoryInformation/Guidances/UCM201283.pd f. Accessed: September 23, 2013.

2. US Food and Drug Administration. Center for Drug Evaluation and Research (CDER). Draft guidance on tacrolimus. December, 2012. http://www.fda.gov/downloads/Drugs/GuidanceCom plianceRegulatoryInformation/Guidances/UCM1810 06.pdf. Accessed: September 23, 2013.

3. US Food and Drug Administration. Center for Drug Evaluation and Research (CDER). Background information for the FDA Meeting of the Advisory Committee for Pharmaceutical Science and Clinical Pharmacology. Topic1: Bioequivalence (BE) and quality standards for narrow therapeutics index (NTI) drug products. July 26, 2011. 
http://www.fda.gov/downloads/AdvisoryCommittees/C ommitteesMeetingMaterials/Drugs/AdvisoryCommittee forPharmaceuticalScienceandClinicalPharmacology/UC M263465.pdf. Accessed; September 23, 2013.

4. Yu LX. Approaches to demonstrate bioequivalence of narrow therapeutic index drugs. Presented at the Meeting of the FDA Advisory Committee for Pharmaceutical Science and Clinical Pharmacology; July 26, 2011; Silver Spring, MD.

http://www.fda.gov/downloads/AdvisoryCommittees/C ommitteesMeetingMaterials/Drugs/AdvisoryCommittee forPharmaceuticalScienceandClinicalPharmacology/UC M266777.pdf. Accessed: September 23, 2013.

5. Dentali F, Donadini MP, Clark N, et al. Brand name versus generic warfarin: a systematic review of the literature. Pharmacotherapy, 2011; 31:384-393.

6. Kesselheim AS, Misono AS, Lee JL, et al. Clinical equivalence of generic and brand-name drugs used in cardiovascular disease - a systematic review and metaanalysis. J Amer Med Assoc. 2008; 300:2514-2526.

7. Vercaigne LM, Zhanel GG. Clinical significance of bioequivalence and interchangeability of narrow therapeutic range drugs: focus on warfarins. J Pharm Pharmaceut Sci. 1998;1:92-94.

8. Health Canada. Guidance document. Comparative bioavailability standards: Formulations used for systemic effects. Ottawa, ON, May 22, 2012. http://www.hc-sc.gc.ca/dhp-mps/prodpharma/applicdemande/guide-ld/bio/gd_standards_ld_normeseng.php. Accessed: September 23, 2013.

9. European Medicines Agency. Committee for Human Medicinal Products (EMA-CHMP). Questions Answers: Positions on specific questions addressed to the pharmacokinetics working party. EMA/618604/2008 Rev. 7. London, February 13, 2013. http://www.ema.europa.eu/docs/en_GB/document libra ry/Scientific guideline/2009/09/WC500002963.pdf.

Accessed: September 23, 2013.

10. Bekersky I, Dressler D, Colburn W, Mekki Q. Bioequivalence of 1 and $5 \mathrm{mg}$ tacrolimus capsules using a replicate study design. J Clin Pharmacol. 1999; 39:1032-1037.

11. Park K, Kim YS, Kwon KI, Park MS, Lee YJ, Kim KH. A randomized, open-label, two-period, crossover bioavailability study of two oral formulations of tacrolimus in healthy Korean adults. Clin Ther. 2007; 29: 154-162.

12. Mathew P, Mandal J, Patel K, et al. Bioequivalence of two tacrolimus formulations under fasting conditions in healthy male subjects. Clin Ther. 2011; 33:1105-1119.

13. Herranz M, Morales-Alcelay S, Corredera-Hernandez $\mathrm{T}$, et al. Bioequivalence between generic tacrolimus products marketed in Spain by adjusted indirect comparison. Int J Clin Pharmacol. 2013; 69: 11571162.

14. Hogan M. Generic tacrolimus: lack of bioequivalence data in transplant recipients raises concerns. Nephrol. Times, 2009; 2:1-15.

15. Tothfalusi L, Endrenyi L, Midha KK, et al. Evaluation of the bioequivalence of highly-variable drugs and drug products. Pharm Res. 2001; 18:728-733.

16. Haidar SH, Davit B, Chen ML, et al. Bioequivalence approaches for highly variable drugs and drug products. Pharm Res. 2008; 25:237-241.

17. Davit BM, Chen M-L, Conner D, et al. Implementation of a reference-scaled average bioequivalence approach for highly variable generic drug products by the US Food and Drug Administration. AAPS J. 2012; 14:914924.

18. Hyslop T, Hsuan F, Holder DJ. A small sample confidence interval approach to assess individual bioequivalence. Stat Med. 2000; 19:2885-2897.

19. Tothfalusi L, Endrenyi L, Garcia AA. Evaluation of bioequivalence for highly variable drugs with scaled average bioequivalence. Clin Pharmacokin. 2009; 48:725-743.

20. Howe WG. Approximate confidence limits on the mean of $\mathrm{X}+\mathrm{Y}$ where $\mathrm{X}$ and $\mathrm{Y}$ are two tabled independent random variables. J Amer Stat Assoc. 1974; 69:789794.

21. Boddy AW, Snikeris FC, Kringle RO, et al. An approach for widening the bioequivalence acceptance limits in the case of highly variable drugs. Pharm Res. 1995; 12:1865-1868.

22. Schuirmann D. A comparison of the two onesided tests procedure and the power approach for assessing the equivalence of average bioavailability. $J$ Pharmacokin Biopharm. 1987; 15:657-680.

23. Midha KK, McKay G, Bialer M, Rawson M. Narrow therapeutic index drugs: an approach to bioequivalence and interchangeability. Presented at the Meeting of the FDA Advisory Committee for Pharmaceutical Science and Clinical Pharmacology; July 26, 2011; Silver Spring, MD. http://www.fda.gov/downloads/AdvisoryCommittees/C ommitteesMeetingMaterials/Drugs/AdvisoryCommittee forPharmaceuticalScienceandClinicalPharmacology/UC M266777.pdf. Accessed: September 23, 2013.

24. Schuirmann DJ. Evaluation of scaling approaches to demonstrate BE of NTI drugs - OGD simulation efforts. Presented at the Meeting of the FDA Advisory Committee for Pharmaceutical Science and Clinical Pharmacology; July 26, 2011; Silver Spring, MD. http://www.fda.gov/downloads/AdvisoryCommittees/C ommitteesMeetingMaterials/Drugs/AdvisoryCommittee forPharmaceuticalScienceandClinicalPharmacology/UC M266777.pdf. Accessed: September 23, 2013.

25. Davit BM. FDA proposal for bioequivalence of generic narrow therapeutic index drugs. Presented at the Meeting of the FDA Advisory Committee for Pharmaceutical Science and Clinical Pharmacology; July 26, 2011; Silver Spring, MD. http://www.fda.gov/downloads/AdvisoryCommittees/C ommitteesMeetingMaterials/Drugs/AdvisoryCommittee forPharmaceuticalScienceandClinicalPharmacology/UC M266777.pdf. Accessed: September 23, 2013.

26. Zhang X, Zheng N, Lionberger RA, Yu LX. Innovative approaches for demonstration of bioequivalence: the 
US FDA perspective. Ther Deliv, 2013; 4:725-740.

27. FDA. Guidance for industry - statistical approaches to establishing bioequivalence. Center for Drug Evaluation and Research, US Food and Drug Administration, Rockville, MD, January, 2001. http:/www.fda.gov/downloads/Drugs/GuidanceCompli anceRegulatoryInformation/Guidances/ucm070244.pdf. Accessed: September 23, 2013..

28. Patnaik RN, Lesko LJ, Chen ML, Williams RL. Individual bioequivalence. New concepts in the statistical assessment of bioequivalence metrics. Clin Pharmacokin. 1997; 33:1-6.

29. FDA. Draft guidance for industry - Bioanalytical method validation, revision 1. Center for Drug Evaluation and Research, US Food and Drug Administration, Rockville, MD, September, 2013. http:/www.fda.gov/downloads/Drugs/GuidanceCompli anceRegulatoryInformation/Guidances/UCM368107.pd f. Accessed: September 23, 2013.

30. European Medicines Agency. Committee for Human Medicinal Products (EMA-CHMP). Guideline on bioanalytical method validation, EMEA/CHMP/EWP/192217/2009, London, July 21, 2011.

http://www.ema.europa.eu/docs/en_GB/document_libra ry/Scientific guideline/2011/08/WC500109686.pdf.

Accessed: September 23, 2013.

31. Kasim NA, Whitehouse M, Ramachandran C, Bermejo $\mathrm{M}$, Lennernäs $\mathrm{H}$, Hussain AS, Junginger $\mathrm{HE}$, et al. Molecular properties of WHO essential drugs and provisional biopharmaceutical classification. Mol Pharmacol. 2004; 12:85-96.

32. Sawoniak AE, Shalansky KF, Zed PJ, Sunderji R. Formulary considerations related to warfarin interchangeability. Can J Hosp Pharm. 2002; 55: 215217.

33. Tothfalusi L, Speidl S, Endrenyi L. Exposure-response analysis reveals that clinically important toxicity difference can exist between bioequivalent carbamazepine tablets. $\mathrm{Br} J$ Clin Pharmacol. 2007; 65:110-122.

34. Tothfalusi L, Endrenyi L. Approvable generic carbamazepine formulations may not be bioequivalent in target patient populations. Int J Clin Pharmacol Ther. 2013, 51(6):525-528.

35. Kudriakova TB, Sirota LA, Rozova GI and Gorkov VA. Autoinduction and steady-state pharmacokinetics of carbamazepine and its major metabolites. Br J Clin Pharmacol. 1992; 33: 611-615.

36. Lionberger R, Jiang $\mathrm{W}$, Huang S-M and Geba G. Confidence in generic drug substitution. Nature Clin Pharmacol Ther. 2013; 94: 438-440.

37. US Food and Drug Administration. Advisory Committee for Pharmaceutical Science and Clinical Pharmacology. Summary minutes of the Advisory Committee for Pharmaceutical Science and Clinical Pharmacology meeting, July 26, 2011. http://www.fda.gov/downloads/AdvisoryCommittees/C ommitteesMeetingMaterials/Drugs/AdvisoryCommittee forPharmaceuticalScienceandClinicalPharmacology/UC M272111.pdf. Accessed: September 23, 2013.

38. Benet LZ, Goyan JE. Bioequivalence and narrow therapeutic index drugs. Pharmacotherapy. 1995; 15:433-440.

39. Boehringer Ingelheim. Comparative bioavailability of dabigatran etexilate (BIBR 1048 MS) formulations. Boehringer Ingelheim International $\mathrm{GmbH}$, Ingelheim, Germany, October 26, 2011.

http://trials.boehringeringelheim.com/content/dam/internet/opu/clinicaltrial/co $\underline{\mathrm{m} \text { EN/results/1160/1160.117 U11-2526-01.pdf. }}$

Accessed: September 23, 2013.

40. European Medicines Agency. Guideline on the investigation of bioequivalence. London, United Kingdom, January 20, 2010.

http://www.ema.europa.eu/docs/en_GB/document_libra ry/Scientific guideline/2010/01/WC500070039.pdf . Accessed: September 23, 2013. 